assessed while the plasma level of VPO1 in patients and the expression of VPO1 in arterial tissues was measured. Cultured human aorta vascular smooth muscle cells were treated with ANGII, and the proliferation activity, VPO1 expression, $\mathrm{H}_{2} \mathrm{O}_{2}$ and HOCL level were examined. The effect of VPO1 RNA interference, apocynin, catalase and PD98059 on VPO1 expression and the proliferation activity of cells were observed.

Results The VPO1 level/expression was significantly increased in patients with essential hypertension and in spontaneously hypertensive rats concomitant with definite vascular remolding by evaluating the intima-media thickness, pressure-strain elastic modulus and stiffness index of carotid artery in patients, as well as the media thickness, lumen diameter, media thickness/lumen diameter ratio and mean nuclear area in artery media in spontaneously hypertensive rats. The angiotension II-stimulated cell proliferation of human aorta smooth muscle cells was inhibited by knockdown of VPO1 using small hairpin RNA. Moreover, the NADPH oxidase inhibitor, apocynin, the hydrogen peroxide scavenger, catalase, but not the ERK1/2 inhibitor, PD98059 attenuated Ang II-mediated upregulation of VPO1 and generation of hypochlorous acid.

Conclusions VPO1 is a novel regulator of vascular smooth muscle cell proliferation via $\mathrm{NADPH}$ oxidase $/ \mathrm{H}_{2} \mathrm{O}_{2} / \mathrm{VPO} 1 / \mathrm{ERK} 1 / 2$ pathways and plays an important role in vascular remodelling during hypertension.

\section{e0595 ANGIOTENSINII MODULATES ION PUMPS OF SMOOTH MUSCLE CELLS DERIVED FROM UMBILICAL ARTERY OF HUMAN NEONATES WITH HYPERTENSIVE FAMILY HISTORY}

doi:10.1136/hrt.2010.208967.595

${ }^{1}$ Qian-Hui SHANG, ${ }^{1} Y$ u FANG, ${ }^{1}$ Qian-feng JIANG, ${ }^{2}$ Jin-Wei LIU. ${ }^{1}$ Institute of Clinical Medine of Zunyi Medical College, Department of Cardiology, Affiliated Hospital of Zunyi Medical College, Zunyi, Guizhou, Chin; ${ }^{2}$ Key Laboratory of Cell Engineering of Guizhou Province, Zunyi, Guizhou, China

Objective To investigate sodium pump and calcium pump activities and mRNA expression level and the changes after AngiotensinII (AngII) treatment in human umbilical artery smooth muscle cells (HUASMCs) isolated from neonates with positive hypertensive family history $\left(\mathrm{FH}^{+}\right)$or with negative hypertensive family history $\left(\mathrm{FH}^{-}\right)$.

Methods Ion pump activities in cultured HUASMCs were detected by spectrophotography. The mRNA expression of sodium pump $\alpha_{1}$-subunit and plasma membrane $\mathrm{Ca}^{2+}$-ATPase isoform 1 (PMCA1) in $\mathrm{FH}^{+}$and $\mathrm{FH}^{-}$HUASMCs was measured by RT-PCR.

Results Sodium pump, calcium pump activities in $\mathrm{FH}^{+}$HUASMCs were higher than those in $\mathrm{FH}^{-}$group $(\mathrm{p}<0.05)$, but the mRNA expression of sodium pump $\alpha_{1}$ subunit and PMCA1 showed no difference between two groups. In $\mathrm{FH}^{-}$group, after 24-h treatment, AngII $\left(1 \times 10^{-7} \mathrm{~mol} / \mathrm{L}\right)$ elevated the activities of sodium pump $(4.62 \pm 0.26$ vs $3.52 \pm 0.33)$ and calcium pump (4.00 \pm 0.31 vs $3.01 \pm 0.32)$, and up-regulated sodium pump $\alpha_{1}$-subunit mRNA expression ( $0.946 \pm 0.099$ vs $0.697 \pm 0.050, \mathrm{n}=5, \mathrm{p}<0.01)$, however higher concentration AngII $\left(1 \times 10^{-6} \mathrm{~mol} / \mathrm{L}\right)$ suppressed the activities of sodium $(2.47 \pm 0.27)$ and calcium pump $(1.79 \pm 0.27)$, and down-regulated sodium pump mRNA expression $(0.445 \pm 0.065)$. Whereas, in $\mathrm{FH}^{+}$groups, both concentration $\left(10^{-6}\right.$ and $\left.10^{-7} \mathrm{~mol} / \mathrm{l}\right)$ of AngII suppressed the activities of sodium pump $(3.49 \pm 0.34$, $2.21 \pm 0.23$ vs $4.70 \pm 0.44)$ and calcium pump $(2.85 \pm 0.31,1.87 \pm 0.16$ vs $4.27 \pm 0.48)$, but only AngII $\left(10^{-7} \mathrm{~mol} / \mathrm{l}\right)$ down-regulated their mRNA expression ( $\alpha_{1}$-subunit: $0.515 \pm 0.133$ vs $0.885 \pm 0.097$, $\mathrm{PMCA}_{1}: 0.165 \pm 0.049$ vs $0.397 \pm 0.046, \mathrm{n}=5, \mathrm{p}<0.01$ ).

Conclusions The activity of sodium pump and calcium pump is increased in $\mathrm{FH}^{+}$HUASMCs. AngII inhibits both $\mathrm{Na}^{+}$and $\mathrm{Ca}^{2+}$ ion pumps activities and mRNA expression in $\mathrm{FH}^{+}$HUASMCs, and may have biphasic effects on ion pump activities and mRNA expression in $\mathrm{FH}^{-}$hUASMCs.

\section{e0596 ROLE OF MONOCYTE CHEMOATTRACTANT PROTEIN-1 ON LARGE ARTERIAL STRUCTURAL AND FUNCTIONAL CHANGE IN PREHYPERTENSIVE SUBJECTS}

doi:10.1136/hrt.2010.208967.596

Wang Shujian, Lu Fanghong, Zhao Yingxin, Liu Zhengdong, Yao Guangtao, Pan Hui. Department of Cardio-cerebro Vascular Control And Research Center

Background and Objects Elevated blood pressure causes a change in vascular remodelling and arterial stiffeness. Dynamic development of the inflammatory reaction may play a role in the early increase of blood pressure. Monocyte chemoattractant protein-1 (MCP-1) which has a chemotactic effect on monocytes/macrophages, is an initial factor of inflammation. However, whether monocyte chemoattractant protein-1 (MCP-1) is altered in the change of large arterial structure and function in prehypertensive subjects has been incompletely investigated.

Method According to the criteria of JNC7, 160 subjects were divided into three groups: (1) normotensive group ( $\mathrm{n}=57)$, (2) prehypertensive group $(n=50)$ and (3) hypertensive group $(n=53)$. Brachium-ankle pulse wave velocity (BaPWV) was measured by an automatic waveform analyser (Form PWV/ABI) and carotid artery intima-media thickness (IMT) was determined ultrasonographically. MCP-1 mRNA level were obtained by real time RT-PCR.

Result In prehypertensives, MCP-1, baPWV and IMT levels are higher than that in normotensives $(p<0.01)$ and lower than that in hypertensives $(p<0.01)$. MCP-1 mRNA level correlated linearly and significantly with baPWV and IMT $(\mathrm{p}<0.01)$, even after adjustments for confounding variables.

Conclusions Large artery remodelling has been found in prehypertensive subjects. PWV and IMT were closely related to the level of blood pressure. MCP-1 may play a role structural and functional vascular changes in prehypertensive subjects.

\section{e0597 SERUM HDL-C LEVELS CORRELATED WITH THE HAEMODYNAMIC AND SEVERITY IN PATIENTS WITH IDIOPATHIC PULMONARY ARTERIAL HYPERTENSION}

doi:10.1136/hrt.2010.208967.597

Zhao Qinhua, Sun Peiyu, Jiang Xin, He Jing, Yang Zijian, Xu Xin, Liu Dong, Yuan Ping, Zhang Rui. Shanghai Pulmonary Hospital

Aims Although dyslipidemia was an established risk factor for cardiovascular disease, its role in the pathogenesis of pulmonary arterial hypertension (PAH) is still unclear. The aims of the current study were to elucidate the clinical significance of the serum dyslipidemia levels in patients with idiopathic PAH (IPAH).

Methods Serum dyslipidemia levels (total-cholesterol (TC), triglyceride (TG), high-density lipoprotein cholesterol (HDL-C), lowdensity lipoprotein cholesterol (LDL-C)) and other clinical datas collected from 90 consecutive adult patients with IPAH from April 2008 to Dec 2009 were retrospectively evaluated in our center. Right heart catheterisation was performed in all patients. Fourty-five age and sex-matched healthy volunteers served as control subjects.

Results The levels of serum TC and HDL-C was significantly decreased in patients with IPAH compared with control subjects $(3.77 \pm 0.86$ vs $4.23 \pm 0.73 \mathrm{mmol} / \mathrm{l}, \quad \mathrm{p}<0.05 ; \quad 1.04 \pm 0.31$ vs $1.46 \pm 0.31 \mathrm{mmol} / 1, \quad \mathrm{p}<0.001$ respectively). Serum HDL-C levels decreased in proportion to the severity of WHO function. Compared with the high HDL-C group, the low HDL-C group demonstrated significantly lower in 6 min walk distance (6MWD), cardiac output 